\title{
PREVENÇÃO DE LITÍASE URINÁRIA A PARTIR DE TERAPIA DE REPOSIÇÃO DE CITRATOS DE POTÁSSIO E MAGNÉSIO: relato de caso
}

\author{
Débora Ribeiro VIEIRA ${ }^{1}$
}

Ronilson Ferreira FREITAS ${ }^{2}$

Dorothea Schmidt FRANÇA ${ }^{3}$

\begin{abstract}
${ }^{1}$ Discente do Curso de Graduação em Medicina e Bolsista do Programa de Iniciação Científica das Faculdades Integradas Pitágoras - FIPMoc. debora_ribeiro_vieira@hotmail.com

${ }^{2}$ Mestre em Saúde, Sociedade e Ambiente pela Universidade Federal dos Vales do Jequitinhonha e Mucuri - UFVJM. Professor das Faculdades Integradas do Norte de Minas - Funorte. ronnypharmacia@gmail.com

${ }^{3}$ Doutora em Ciências Biológicas (Fisiologia e Farmacologia) pela Universidade Federal de Minas Gerais - UFMG. Professora do Centro de Ciências Biológicas e da Saúde da Universidade Estadual de Montes Claros - Unimontes e das Faculdades Integradas Pitágoras de Montes Claros - FIPMoc. dorotheafranca@ gmail.com
\end{abstract}

Recebido em: 06/10/2016 - Aprovado em: 09/01/2017 - Disponibilizado em: 01/07/2017

\begin{abstract}
RESUMO:
As doenças renais acometem milhares de pessoas com faixa etária economicamente ativa em todo o mundo, gerando danos à economia e tornando-se um grande problema de saúde pública, devido à elevada recorrência e alto índice de complicações, o que gera elevados custos ao sistema de saúde. Independente da etiologia da doença de base, a perda da função renal acarreta desfechos indesejados que podem ser prevenidos ou retardados. A litíase urinária ocupa o terceiro lugar entre as afecções mais frequentes do trato urinário e é definida como a presença de um ou mais cálculos no interior de órgãos ou canais do sistema urinário, desde os cálices renais até a bexiga. Alterações metabólicas como hipercalciúria, hiperucosúria, hipocitratúria, hiperoxalúria, cistinúria e infecção urinária são as principais causas da formação dos cálculos e por isso é importante identifica-las precocemente para não realizar um diagnóstico tardio. Neste sentido, este estudo teve como objetivo relatar o caso de uma paciente com hipercalciúria, hipocitratúria e litíase urinária em que o uso de terapia de reposição de citratos de potássio e magnésio foi importante escolha para a prevenção da formação de litíase renal. A litíase cálcica representa $85 \%$ do total de casos e ocorre devido ao aumento do cálcio, ácido úrico ou oxalato urinário e/ou diminuição do citrato urinário. A combinação do aumento do filtrado de cálcio e a diminuição da reabsorção tubular desse íon conduz inevitavelmente a hipercalciúria. Atualmente a suplementação de citrato de potássio e magnésio, que são substâncias capazes de inibir esse processo de cristalização, tem sido muito comercializadas. O citrato tem dois efeitos importantes na prevenção da nefrolitíase devido aos efeitos solubilizante e inibidor da cristalização dos sais de cálcio. Assim, é possível concluir que, como a hipercalciúria e a hipocitratúria são os principais fatores de risco para a litíase renal, a reposição desses íons é de grande importância na prevenção de litíases e da perda da função renal.
\end{abstract}

Palavras-chave: Nefrolitíase. Hipercalciuria. Hipocitratúria.

\section{PREVENTION OF URINARY LITHIASIS WITH POTASSIUM AND MAGNESIUM CITRATES: case report}

\begin{abstract}
Kidney diseases affect thousands of people economically active age group worldwide, causing damage to the economy and becoming a major public health problem due to the high recurrence and high complication rate, which generates high costs to the system Cheers. Regardless of the etiology of the underlying disease, the loss of renal function leads to unwanted outcomes that can be prevented or delayed. The urolithiasis ranks third among the most common disorders of the urinary tract and is defined as the presence of one or more calculations on the inside of organs or channels of the urinary system, from the kidney to the bladder goblets. metabolic abnormalities such as hypercalciuria, hiperucosúria, hypocitraturia, hyperoxaluria, cystinuria and urinary tract infection are the main causes of the formation of calculations and so it is important to identify them early to not hold a late diagnosis. Thus, this study aimed to report the case of a patient with hypercalciuria, hypocitraturia and urolithiasis in the replacement therapy use of potassium citrate and magnesium was important choice for the prevention of kidney stones. The calcium lithiasis represents $85 \%$ of all cases
\end{abstract}


and occurs because of the increased calcium, uric acid or urinary oxalate and/or decrease in urinary citrate. The combination of increased calcium filtered and reduced tubular reabsorption of this ion leads inevitably hypercalciuria. Currently, potassium and magnesium citrate supplementation, which are substances capable of inhibiting the process of crystallization, has long been marketed. Citrate has two important effects in the prevention of nephrolithiasis due to the solubilizing effects and inhibitor of crystallisation of calcium salts. Thus, we conclude that, as hypercalciuria and hypocitraturia are the main risk factors for nephrolithiasis, the replacement of these ions is of great importance in the prevention of urolithiasis and loss of kidney function.

Keywords: Nephrolithiasis. Hypercalciuria. Hypocitraturia.

\section{INTRODUÇÃO}

Litíase, urolitíase, nefrolitíase, cálculo ou calculose urinária é a presença de um ou mais cálculos no interior de órgãos ou canais do sistema urinário, desde os cálices renais até a bexiga, ou seja, é a formação de conglomerados cristalinos e de matriz orgânica que podem apresentar tamanhos variados capazes de causar sintomas e crises álgicas de alta prevalência e recorrência, que são de grande custo para o sistema de saúde (RIELLA, 2010; CÉSAR et al., 2012).

Segundo a Sociedade Brasileira de Urologia (2014), a urolitíase ocupa o terceiro lugar entre as afecções mais frequentes do trato urinário. Ocorre três vezes mais entre homens, com maior incidência na faixa etária entre 30 a 50 anos.

Segundo Riella (2010), os cálculos urinários podem ser resultantes do excesso de solutos que promovem a supersaturação ou cristalização da urina, pela diminuição da ingestão hídrica, o que dificulta a diluição dos solutos, possibilitando a cristalização desses minerais. A composição desses cálculos pode ser por oxalato de cálcio puro, oxalato de cálcio e fosfato, fosfato de cálcio puro, estruvita (formado de fosfato amoníaco magnesiano), ácido úrico ou cistina; sendo os compostos por oxalatos mais frequentes, somados sendo responsáveis por quase $70 \%$ das taxas de incidência (PERES et al 2011; CÉSAR et al., 2012; GIORDANO et al., 2014).

Este trabalho teve como objetivo relatar o caso de uma paciente com hipercalciúria, hipocitratúria e litíase urinária em que o uso de terapia de reposição de citratos de potássio e magnésio foi importante escolha para a prevenção da formação de litíase renal.

\section{RELATO DE CASO}

Paciente MSRV, 47 anos, casada, G5P2A3, relata crises nefríticas, caracterizadas por dores em flanco e dorso, intensas, contínuas, que acompanhavam náuseas, vômitos e calafrios e sudorese desde os dezoito anos de idade, devido à formação de cálculos renais originados de hipercalcemia sérica. Apresenta histórico de internações frequentes desde a manifestação da primeira crise com cálculos expelidos desde então e infecções urinárias de repetição, 
porém, sem diagnóstico definitivo de um fator causal.

Em 2003, aos 35 anos, apresentou o quadro obstrutivo mais exuberante, com presença de litíase em canal de ureter que acarretou sepse grave, necessitando cirurgia de emergência.

Em 2007, aos 39 anos, através de testes metabólicos da urina, a paciente foi diagnosticada com hipocitratúria endógena com déficit de citrato de potássio e de magnésio, minerais importantes na inibição da formação de cálculos de oxalato de cálcio. Essa deficiência endógena justifica a hipercalciúria presente desde os dezoito anos, o que propicia a formação de litíases urinárias. A paciente foi orientada a mudanças alimentares e foram prescritos suplementos de citrato de potássio e magnésio em baixas doses.

Em 2011, aos 43 anos manifestou-se outro caso grave que precisou de intervenção cirúrgica de urgência, necessitando-se assim de elevação das doses de suplementação dos citratos de potássio e magnésio.

Atualmente a paciente realiza acompanhamento semestral com avaliação da função metabólica do cálcio, ácido úrico e citratos, com objetivo de detecção precoce de formação de litíases urinárias.

\section{DISCUSSÃO}

A litíase tem atingido o homem desde a antiguidade. Indícios da patologia foram encontrados em múmias egípcias. É uma patologia heterogênea de recorrência elevada e com tendência à cronicidade. É um problema de saúde pública devido à sua terapêutica, na maioria das vezes, ser cirúrgica e sua cronicidade ocasionar insuficiência da função renal, doença que afeta diretamente a qualidade de vida do indivíduo, que necessita de um tratamento contínuo por um longo período (CASTRO et al., 2003). Um programa de rastreamento de fatores de risco e de prevenção eficaz pode reduzir a incidência da doença em $80 \%$ como relatado por Gomes et al., (2005). Segundo a Sociedade Brasileira de Urologia (2014) e Peres et al., (2011), a litíase urinária acomete cerca de 5 a $15 \%$ da população e a recidiva após a primeira crise é de $50 \%$ em cinco anos.

Alterações metabólicas como hipercalciúria, hiperucosúria, hipocitratúria, hiperoxalúria, cistinúria e infecção urinária são as principais causas da formação dos cálculos e por isso é importante identifica-las precocemente para diagnosticar a litíase renal (PERES et al., 2011).

Vários fatores etiopatogênicos e epidemiológicos interferem na formação de novos cálculos como por exemplo o tipo de ocupação, sexo, idade, sedentarismo, obesidade, clima e malformações do sistema 
urinário, dieta de alto teor de proteínas e sal e baixa ingestão de hídrica. Fatores genéticos são importantes, podemos encontrar vários casos numa mesma família (SOCIEDADE BRASILEIRA DE UROLOGIA, 2014; MURTINHO; PEDRO, 2014).

A influência da dieta nas alterações metabólicas dando consequência â formação dos cálculos urinários tem sido muito evidenciada (PERES et al., 2011).

A suplementação de citrato de potássio e magnésio, que são substâncias capazes de inibir esse processo de cristalização, tem sido muito prescritas e comercializadas. Os citratos tem dois efeitos importantes na prevenção da litíase devido aos efeitos solubilizantes e inibidores da cristalização dos sais de cálcio, são livremente filtrados e excretados pelos rins (SAKUNO et al., 2002).

Além da suplementação de citratos, hábitos diários e pessoais devem ser aconselhados e estimulados a todos os indivíduos como o aumento da ingestão hídrica, para a diminuição da concentração de componentes litiásicos na urina, e o incentivo à alimentação equilibrada, aumentando o consumo de magnésio, de frutas frescas e fibras provenientes de cereais integrais e diminuição do consumo de proteínas e sódio. O acompanhamento alimentar é muito importante uma vez que esse problema, além de comprometer a qualidade de vida do indivíduo, pode ter como consequência a perda irreversível da função renal (NERBASS, 2014)

\section{CONCLUSÃO}

Como a hipercalciúria e a hipocitratúria são os principais fatores de risco para a litíase renal, a reposição desses íons é de grande importância na prevenção de litíases e da perda da função renal. A avaliação do perfil metabólico desses íons é de fácil acesso e facilita a identificação do risco de formação de cálculos urinários e podem auxiliar no planejamento e estabelecimento de estratégias para tratamentos clínicos mais eficazes, além da implementação de medidas direcionadas à prevenção de litíases renais em pacientes de risco ou que manifestam formação de litíases e infecções de urina de repetição.

\section{REFERÊNCIAS}

CASTRO, M.; CAIUBY, A. V. S.; DRAIBE, S. A.; CANZIANI, M. E. F. Qualidade de vida de pacientes com insuficiência renal crônica em hemodiálise avaliada através do instrumento genérico SF-36. Rev Assoc Med Bras. v. 49, n. 3, p. 245-9, 2003.

CÉSAR, F. O.; CASTRO, F. G.; RODRIGUES, M. L. D.; SOUSA, M. F. Avaliação de fatores de risco para litíase urinária em Goiânia. Faculdade de Medicina da Universidade Federal De Goiás (FM/UFG), 2012. Disponível em: < http://www.sbpcnet.org.br/livro/63ra/conpeex 
/pibic/trabalhos/FERNA000.PDF> Acesso

em: 05 de outubro de 2015.

GORDIANO, E. A.; TONDIN, L. M.;

MIRANDA, R. C.; BAPTISTA, D. R.;

CARVALHO, M. Evaluation of food intake and excretion of metabolites in

nephrolithiasis. J Bras Nefrol. v. 36, n. 4, p.

437-445, 2014.

GOMES, P. N.; CABRITA, M.;

RODRIGUES, M.; VEJA, P.; COUTINHO, A.; ROSA, G.; NEVES, J. Profilaxia da litíase renal. Acta Urológica. v. 22, n. 3, p. 47-56, 2005.

MURTINHO, V. C.; PEDRO, L. S. Abordagem diagnóstica e terapêutica da cólica renal por litíase urinária. FMUC, 2014. Disponível em: < https://estudogeral.sib.uc.pt/bitstream/10316/2 6282/2/TrabalhoFinal_VeraPedro.pdf $>$. Acesso em: 05 de outubro de 2015.

NERBASS, F. B. Orientação dietética e litíase renal. Jornal Brasileiro de Nefrologia. v. 36, n. 4 , p. $428-429,2014$.

PERES, L. A. B.; ALMEIDA, L. P.; BOLSON, L. B.; BRITES, M. F.; DAVID, J. M.; TAZIMA, L. Investigation of nephrolithiasis in the West of Paraná. Jornal Brasileiro de Nefrologia. v. 33, n. 2, p. 160165, 2011.

RIELLA, M. C. Princípios de Nefrologia e Distúrbios Hidroeletrolíticos. 5 ed. Rio de Janeiro: Guanabara Koogan, 2010.

SOCIEDADE BRASILEIRA DE UROLOGIA - Secção São Paulo. Diretrizes de Urologia. Disponível em: <www.sbusp.org.br>. Acesso em: 05 de outubro de 2015.

SAKUNO, M. L. D.; TAKAHACHI, G.; MARTINS, A. B. T.; JANEIRO, V.; BOFETTI, R.; TABUTI, E. K.; BARBIERI, T. M. Citrato urinário na nefrolitíase. Revista Brasileira de Análises Clínicas. v. 34, n. 3, p. 169-171, 2002. 